\title{
Design of an L-Band Cross-Dipole Phased Array Feed for FAST
}

\author{
Yubing Han and Luoqing Zhong \\ Department of Electronic and Optical Engineering, Nanjing University of Science and Technology, Nanjing 210094, China \\ Correspondence should be addressed to Yubing Han; hanyb@mail.njust.edu.cn
}

Received 22 March 2016; Accepted 20 April 2016

Academic Editor: Herve Aubert

Copyright (C) 2016 Y. Han and L. Zhong. This is an open access article distributed under the Creative Commons Attribution License, which permits unrestricted use, distribution, and reproduction in any medium, provided the original work is properly cited.

\begin{abstract}
The Five-Hundred-Meter Aperture Spherical Telescope (FAST) is a Chinese megascience project that aims to build the largest single dish radio telescope in the world. Given its multiple simultaneous beam formation, phased array feed (PAF) is widely used to extend the field of view and enhance the survey speed of the radio telescope. In this study, a broadband and low cost PAF element using cross-dipole antenna at L-band is designed based on the requirement of the FAST. The antenna is fed by two microstrip baluns which have high performance and is easy to manufacture compared to the traditional coaxial balun. A simple system model is also introduced to evaluate the PAF performance. The measured results of the fabricated element and the simulations of the system performance validate the effectiveness of element design.
\end{abstract}

\section{Introduction}

Observation at radio wavelength has acquired a great scientific and applied importance in the field of astronomy. Many new types of astronomical object have been discovered by radio astronomical methods, including quasars, pulsars, and cosmic microwave background [1]. In the field of modern astronomy, high sensitivity and resolution are the key requirements. A novel method for enhancing the performance of reflector telescopes is to employ a phased array feed (PAF). Compared with traditional cluster horn feeds, PAFs can provide steerable beams over a continuous field of view to increase survey speed. This method increases sensitivity and provides a means to mitigate radio frequency interference. Currently several international groups employ PAFs for radio astronomy. In Canada, the Dominion Radio Astrophysical Observatory (DRAO) has been developing Vivaldi arrays for the PHased Array Demonstrator (PHAD) system [2]. The Australian Commonwealth Scientific and Industrial Research Organization (CSIRO) has designed a square patch array for the Australian Square Kilometer Array Pathfinder (ASKAP) project [3]. The Brigham Young University (BYU) radio astronomy group has been working with the National Radio Astronomy Observatory (NRAO) to develop PAFs on NRAO's 20-meter dish in Green Bank, West Virginia [4].
As one of the megascience facilities for basic research in China, the current largest single dish radio telescope, the Five-Hundred-Meter Aperture Spherical Telescope (FAST), is being constructed in a karst depression in Guizhou province [5]. Preliminary performance parameters for FAST are shown in Table 1. Figure 1 shows the geometric model of the reflector which is combined of paraboloid and spherical regions [6]. Nine groups of feed working together with the receiver cover a frequency range from $70 \mathrm{MHz}$ to $3 \mathrm{GHz}$. According to the performance specification of L-band PAF in Table 2, a broadband cross-dipole array is designed in this paper.

\section{Phased Array Feed System}

The system model of the FAST is shown in Figure 2. Incident signals from the universe are reflected by the reflector and received by the PAFs placed at the focal point of the reflector dish. Thereafter, the received signals are transmitted to a low noise amplifier (LNA). Through downconversion, filter, and sampling, signals are transferred to the beamforming network. The beamformer weighs the voltages at the receiver outputs and adds them to generate the desired receiving pattern. 


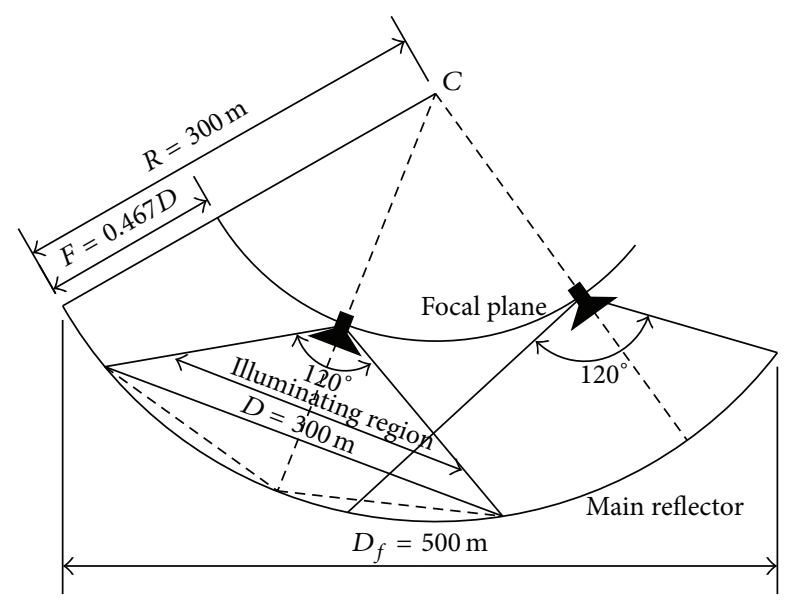

FIGURE 1: Geometrical structure of the FAST.

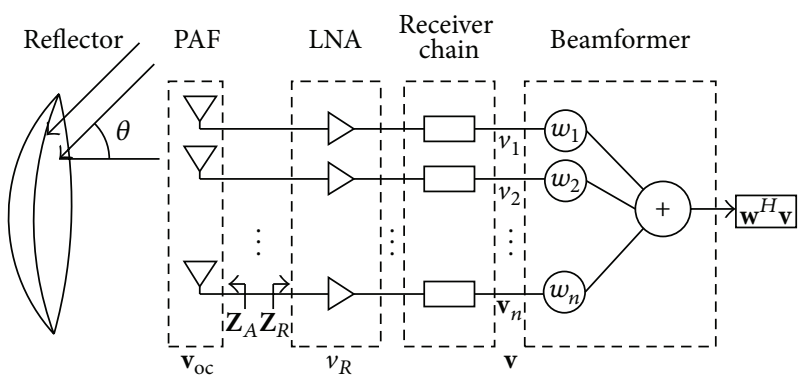

Figure 2: System diagram from arrays to beamformer.

TABLE 1: Performance specifications for FAST.

\begin{tabular}{lc}
\hline Parameter & Quantity \\
\hline Aperture & $500 \mathrm{~m}$ \\
Opening angle & $120 \mathrm{deg}$ \\
Illuminated aperture & $300 \mathrm{~m}$ \\
Focal length & $140.1 \mathrm{~m}$ \\
Zenith angle & $50 \mathrm{deg}$ \\
\hline
\end{tabular}

TABLE 2: Performance specification for L-band FAST-PAF.

\begin{tabular}{lc}
\hline Parameter & Quantity \\
\hline Operating frequency range & $1.05 \mathrm{GHz}-1.45 \mathrm{GHz}$ \\
Instantaneous bandwidth & $\geq 500 \mathrm{MHz}$ \\
Polarization & Dual-polarization \\
Field of view & $0.6 * 0.6 \mathrm{deg}^{2}$ \\
Simultaneous beams & $>100$ \\
System noise temperature & $\leq 25 \mathrm{~K}$ \\
Sensitivity & $\geq 2000$ \\
Aperture efficiency & $\geq 55 \%$ (in $300 \mathrm{~m}$ diameter) \\
Radiation efficiency & $\geq 98 \%$ \\
\hline
\end{tabular}

Assume that $\mathbf{Z}_{A}$ is the array impedance matrix and $\mathbf{Z}_{R}$ is the LNA impedance matrix. The relationship between the output voltages of the receiver and antenna array is shown as follows:

$$
\mathbf{v}=g \mathbf{Z}_{R}\left(\mathbf{Z}_{R}+\mathbf{Z}_{A}\right)^{-1} \mathbf{v}_{\mathrm{oc}}
$$

where $g$ is the gain of the receiver and $\mathbf{v}_{\mathrm{oc}}$ is the opencircuit voltage of the array. The receiver output voltages $\mathbf{v}$ can be expressed in terms of signal, noise, and interference components [7]:

$$
\mathbf{v}=\left[v_{1}, v_{2}, \ldots, v_{N}\right]^{T}=\mathbf{v}_{\text {sig }}+\mathbf{v}_{\text {int }}+\mathbf{v}_{n},
$$

where $\mathbf{v}_{\text {sig }}$ is a vector of voltages associated with the signal of interest, $\mathbf{v}_{\text {int }}$ is the voltage associated with interference, and $\mathbf{v}_{n}$ is the noise voltage. Each component is independent of the others.

The signal covariance matrix can be expressed as

$$
\begin{aligned}
\mathbf{R}_{v} & =E\left[\mathbf{v} \mathbf{v}^{H}\right]=E\left[\mathbf{v}_{\text {sig }} \mathbf{v}_{\text {sig }}^{H}\right]+E\left[\mathbf{v}_{\text {int }} \mathbf{v}_{\text {int }}^{H}\right]+E\left[\mathbf{v}_{n} \mathbf{v}_{n}^{H}\right] \\
& =\mathbf{R}_{\text {sig }}+\mathbf{R}_{\text {int }}+\mathbf{R}_{n},
\end{aligned}
$$

where $\mathbf{R}_{\text {sig }}$ is the correlation matrix of the signal of interest, $\mathbf{R}_{\text {int }}$ is the correlation matrix of the voltage with interference, and $\mathbf{R}_{n}$ is the correlation matrix from the noise voltage.

The beamformer output voltage is

$$
y=\mathbf{w}^{H} \mathbf{v}
$$

where $\mathbf{w}$ represents the beamforming weight vectors. The output SNR of the system is defined as

$$
\mathrm{SNR}=\frac{\mathbf{w}^{H} \mathbf{R}_{\mathrm{sig}} \mathbf{w}}{\mathbf{w}^{H} \mathbf{R}_{n} \mathbf{w}} .
$$

The most common beamformer is the max-SNR beamformer whose weight is [8]

$$
\mathbf{w}=\mathbf{R}_{n}^{-1} \mathbf{a}(\theta),
$$

where $\mathbf{a}(\theta)$ is the steering vector of the desired signal.

Among all figures of merit for phased arrays in radio astronomy, aperture efficiency $\eta_{\text {ap }}$, system temperature $T_{\text {sys }}$, and sensitivity $A_{e} / T_{\text {sys }}$ are the most significant.

Aperture efficiency is a dimensionless parameter that measures how close the antenna comes to using all the radio power that enters its physical aperture. The aperture efficiency for a lossless antenna is defined as the ratio of the effective area $A_{e}$ to the physical area $A_{\text {phys }}$ of the aperture and is computed as [9]

$$
\eta_{\text {ap }}=\frac{A_{e}}{A_{\text {phys }}}=\frac{k_{b} T_{\text {iso }} B}{S^{s i g} A_{\text {phys }}} \frac{\mathbf{w}^{H} \mathbf{R}_{\text {sig }} \mathbf{w}}{\mathbf{R}_{\text {iso }} \mathbf{w}},
$$

where $k_{b}$ is Boltzmann's constant, $B$ is the system bandwidth, $S^{\text {sig }}$ is the incident power density of the antenna terminals, $T_{\text {iso }}$ is the noise temperature resulting from an ideal isotropic thermal noise environment, and $\mathbf{R}_{\text {iso }}$ is the correlation matrix from the isotropic noise.

System temperature $T_{\text {sys }}$ is written as the ratio of beamformed noise contributions [10]:

$$
T_{\text {sys }}=T_{\text {iso }} \frac{P_{\text {noise }}}{P_{\text {iso }}}=T_{\text {iso }} \frac{\mathbf{w}^{H} \mathbf{R}_{n} \mathbf{w}}{\mathbf{w}^{H} \mathbf{R}_{\text {iso }} \mathbf{w}},
$$

where $P_{\text {noise }}=\mathbf{w}^{H} \mathbf{R}_{n} \mathbf{w}$ and $P_{\text {iso }}=\mathbf{w}^{H} \mathbf{R}_{\text {iso }} \mathbf{w}$. 


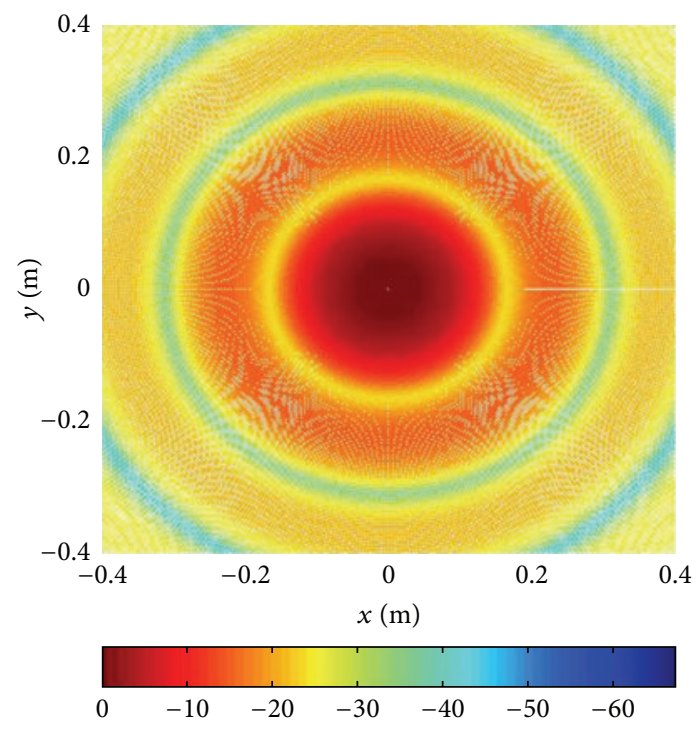

(a)

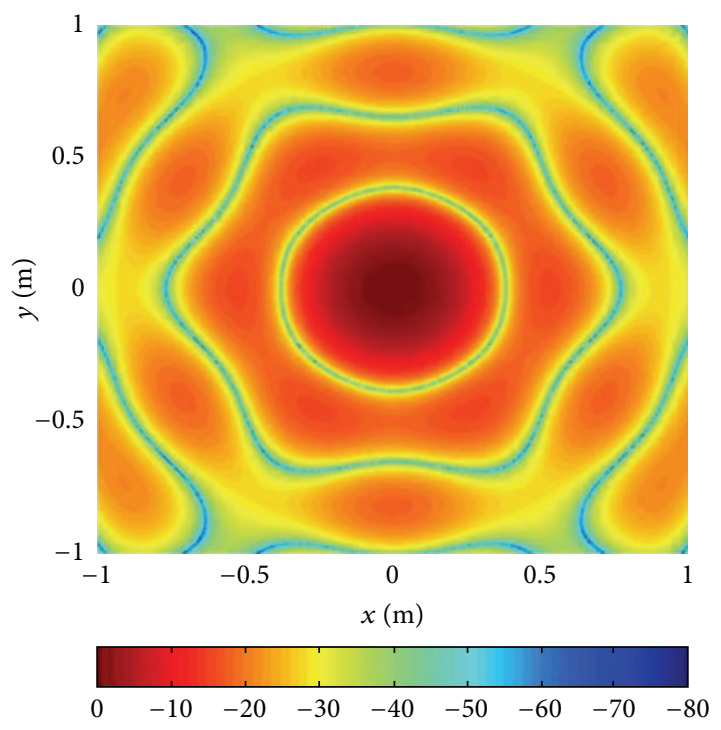

(b)

FIGURE 3: Focal field distribution and radiation pattern in the focal plane. (a) Focal field distribution with vertical incident plane wave. (b) Radiation pattern of 37-element hexagonal array.

Sensitivity is defined as the effective receiving area relative to the system noise temperature. A higher ratio means that weaker signals can be detected. In terms of antenna parameters, sensitivity can be given as [11]

$$
S=\frac{A_{e}}{T_{\text {sys }}}=\frac{k_{b} B}{S^{\text {sig }}} \frac{\mathbf{w}^{H} \mathbf{R}_{\text {sig }} \mathbf{w}}{\mathbf{w}^{H} \mathbf{R}_{n} \mathbf{w}}=\frac{k_{b} B}{S^{\text {sig }}} \mathrm{SNR} .
$$

As can be seen from (9), the max-SNR beamformer can directly maximize the signal-to-noise ratio (SNR), which is equivalent to maximizing sensitivity [12]. To increase sensitivity, the effective area of the reflective surface is broadened or the temperature of system noise is decreased.

\section{PAF Configuration}

The $3 \mathrm{~dB}$ beam width of the reflection surface is defined as

$$
\mathrm{HPBW}=70^{\circ} \frac{\lambda}{D}
$$

where $D$ is the aperture of the reflector and $\lambda$ is the wavelength. The half-power beam width (HPBW) of the FAST is $0.056^{\circ}$. Discussion in the later sections uses HPBW as the measurement scale of the field of view.

3.1. Array Formation. Focal field is defined as the electric and magnetic field distribution from an incident wave in the focal region of the reflector. To achieve maximum energy, the aperture field of the antenna array should match the focal field. Figure 3(a) shows the focal field distribution caused by a plane wave incident to the vertical direction using the reflector parameters of the FAST.

PAF is mounted on the focal plane to restore the information of field distribution. Reference [13] proves that hexagonal sampling is an optimal sampling strategy for the band-limited signal in a circular area in the plane. Assume that $n$ is the number of rings of the array (the central element is the first circle), and element spacing is half wavelength. The radiation pattern of a hexagonal array which is composed of ideal omnidirectional antennas is [14]

$$
\begin{aligned}
B_{u}\left(u_{x}, u_{y}\right) & =\frac{1}{N} \\
\cdot & \sum_{i=-n / 2}^{n / 2} \exp \left\{j \pi\left[i \frac{\sqrt{3}}{2} u_{y}-\frac{n-|i|-1}{2} u_{x}\right]\right\} \\
& \cdot \sum_{j=0}^{n-|j|-1} \exp \left\{j \pi n u_{x}\right\},
\end{aligned}
$$

where $N=3 n^{2}-3 n+1$ is the total element number, $u_{x}=$ $\sin \theta \cos \phi, u_{y}=\sin \theta \sin \phi, \theta$ is the elevation angle, and $\phi$ is the azimuth angle.

Figure 3(b) shows the radiation pattern of the 37-element hexagonal array with equal-amplitude excitation. The figure clearly shows that the radiation pattern from hexagonal array has sixfold symmetry which is very suitable for PAFs. Compared with a rectangular array, a hexagonal arrangement can achieve the same gain and sensitivity with fewer elements, which reduces the calculation of the beamformer [6]. Therefore, hexagon is chosen as the array arrangement of the FAST.

3.2. Array Size. Given that the phase center of the PAF horizontally deviates from the focal point, the focal field changes and causes the beam to be deflected by an angle from the boresight axis. 


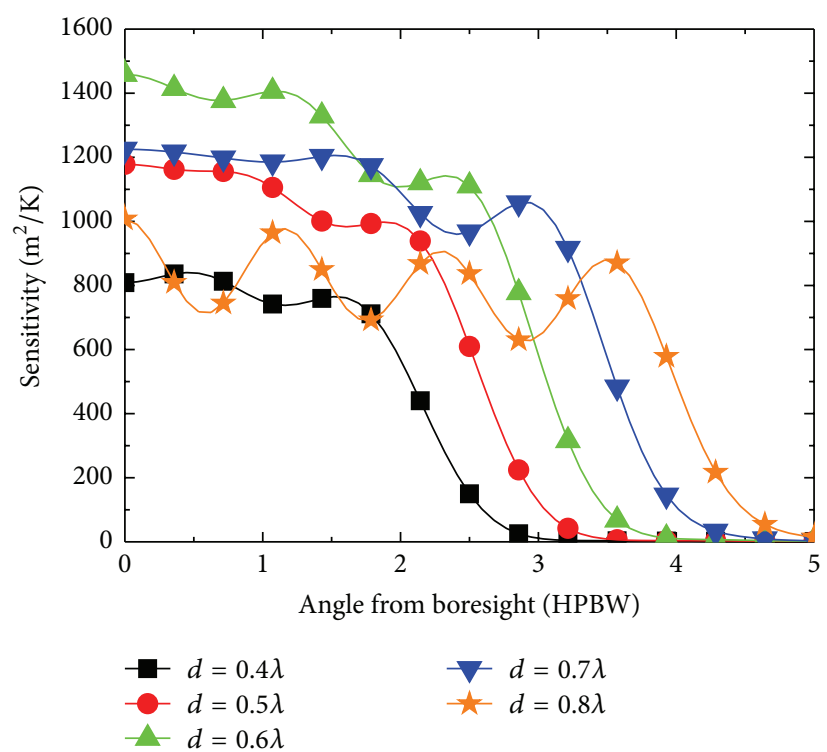

(a)

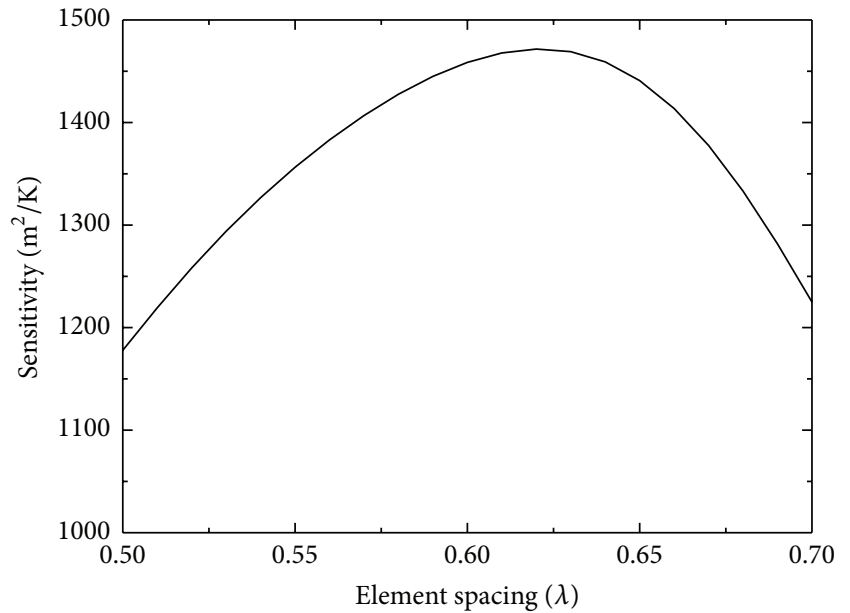

(b)

FIGURE 4: Relationship between sensitivity and element spacing. (a) Sensitivity variation with the increase of spacing. (b) Sensitivity of boresight over different element spacing.

Beam deviation factor is defined as [15]

$$
\mathrm{BDF}=\frac{\theta_{b}}{\theta_{f}}=\sin ^{-1}\left[\frac{d}{f} \frac{1+k(D / 4 f)^{2}}{1+(D / 4 f)^{2}}\right] \frac{1}{\tan ^{-1}(d / f)},
$$

where $\theta_{b}$ is the angle from the focal point to the main beam, $\theta_{f}$ is the angle from the focal point to the feed element, $f$ is the focal length, $d$ is the offset distance of the feed, and $k$ is a coefficient related to $f$ and $d$.

Due to $d \ll f$, (12) can be rewritten as

$$
\mathrm{BDF} \approx \frac{1+k(D / 4 f)^{2}}{1+(D / 4 f)^{2}} .
$$

Given the maximum scan angle $\theta_{b, \max }$, the distance between the phase center of PAF and the focal point can be estimated as follows:

$$
d_{\max }=\tan \left(\frac{\theta_{b, \max }}{\mathrm{BDF}}\right) f
$$

According to [16], when the incoming plane wave is uniform and on the boresight axis, the primary polarization of the focal field is

$$
E_{\mathrm{co}}=2 E_{0} \frac{\pi D^{2}}{4 f \lambda} \frac{J_{1}(u)}{u}
$$

where $E_{0}$ is the amplitude of the incident field, $D$ is the diameter of the reflecting surface, $u=k r \sin \theta_{0}, r$ is the distance from the reference point to the focal point, $\theta_{0}$ is the angle of the reflection, and $J_{1}(x)$ is the first kind of Bessel function.

As shown in Figure 3(a), energy is concentrated near the focal point which forms different rings and zero depressions.
About 95\% energy can be received when the array covers an area with an edge in the third zero depression.

The distance between the focal point and the third zero depression can be obtained from (15) as follows:

$$
d_{3 \text { null }}=\frac{1.62 \lambda}{\sin \theta_{0}} .
$$

Taking into account the defocusing effect and the decrease of array gain, the diameter of the PAF which meets the requirements of scanning can be calculated by

$$
R_{\text {paf }} \geq d_{\text {max }}+d_{3 \text { null }} .
$$

The minimum radius can be obtained by substituting $\theta_{0}=$ $120^{\circ}, D=300 \mathrm{~m}, f=300 \times 0.46 \mathrm{~m}, \theta_{b, \max }=0.3^{\circ}, \lambda=0.24 \mathrm{~m}$, and $k=0.4$ into corresponding parameters:

$$
R_{\text {paf,min }}=1.3 \mathrm{~m} \text {. }
$$

3.3. Element Spacing. Information on focal field distribution can be obtained through focal sampling of the elements of the array. The maximum spacing that satisfies the sampling theorem is [16]

$$
d_{s}=\frac{1}{0.7 \pi \sin \theta_{0}}
$$

and when the hexagonal array is applied, the element spacing can be increased. The sampling interval can keep expanding when the focal diameter of the reflecting surface is relatively large.

In order to determine the optimal array element spacing, a 37-element array which is composed of ideal dipole antennas is designed. Figure 4(a) shows the sensitivity variation with the increase of element spacing. It can be seen that 


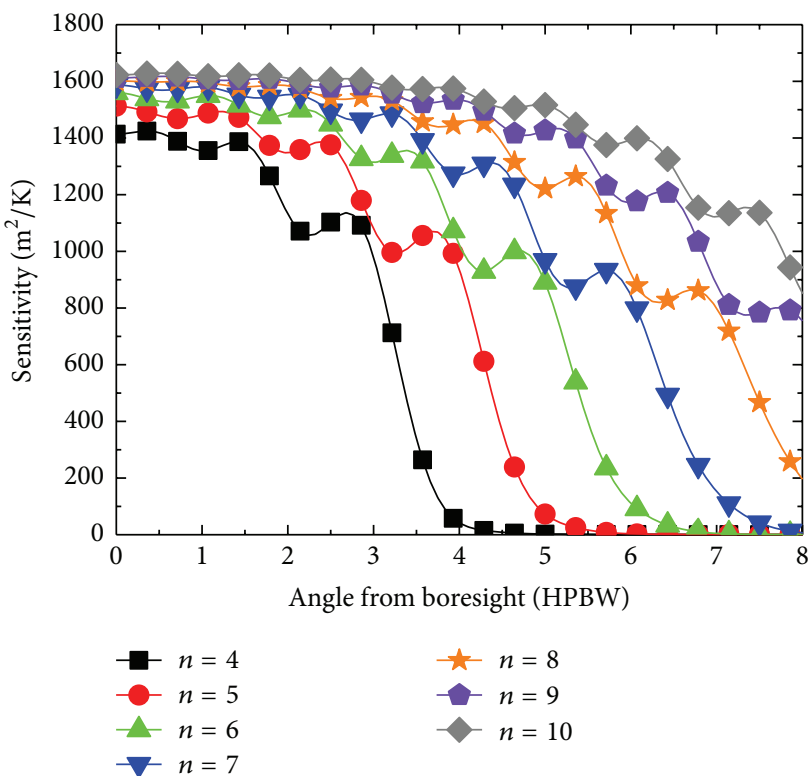

(a)

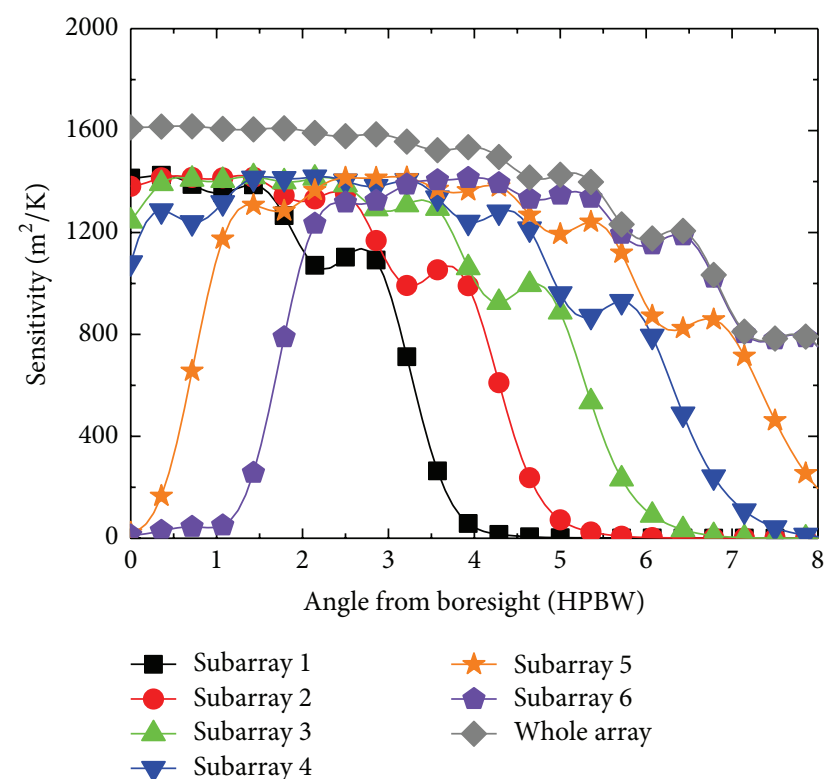

(b)

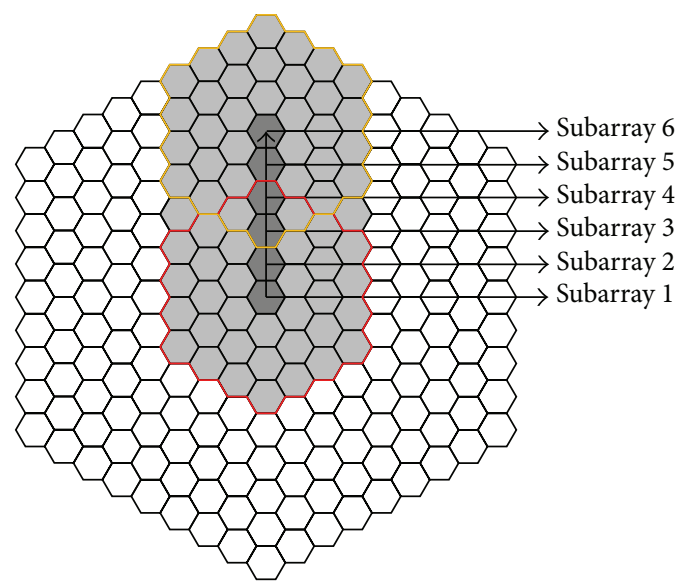

(c)

FIGURE 5: Sensitivity versus different numbers of elements and different subarrays. (a) Sensitivity with different number of feeds. (b) Sensitivity with six 37-element subarrays at different locations and (c) six 37-element subarrays from center to the array edge.

when the element spacing is larger, the array can receive more energy from a wider scan angle. However, the gain and sensitivity of the antenna are reduced because of the discontinuity of the receiving aperture. As the element spacing becomes smaller, the ability of the receiving energy from the directions near the boresight is enhanced, but the field of view becomes narrow. If the element spacing is too small, mutual coupling becomes strong and can result in the worsening of the sensitivity curve. Thus, there exists an optimum spacing which can balance the sensitivity and the field of view. Considering that the sensitivity reaches the peak when element spacing is in the range of $0.5 \lambda-0.7 \lambda$, sensitivity of boresight over different element spacing is simulated in Figure 4(b).

As shown in Figure 4(b), the sensitivity arrives to the top when element spacing is $0.63 \lambda$. However, in order to broaden the field of view, the optimum spacing should be a little larger than $0.63 \lambda$. On balance, $0.65 \lambda$ is selected as the element spacing.

3.4. Number of Elements. Based on the conclusion above, the minimum radius of the array is $1.3 \mathrm{~m}$ and element spacing is $0.65 \lambda$. The central frequency of the FAST in L-band is $1.25 \mathrm{GHz}$. If hexagon is chosen as the array arrangement, $n$ can be calculated as

$$
n=\frac{1.3}{0.65 \lambda}+1=9.33 \approx 9 .
$$

Figure 5(a) shows the sensitivity improvement over field of view with increasing elements at $1.25 \mathrm{GHz}$. If the edge of the field of view is defined as $1 \mathrm{~dB}$ or $20 \%$ maximum acceptable sensitivity loss position [6], 9 or more rings of feeds $(n \geq$ 9) should be employed to achieve the requirement of $0.6 *$ $0.6 \mathrm{deg}^{2}$ field of view, which equals $5.36 * 5.36 \mathrm{HPBW}^{2}$. Taking 
TABLE 3: Summary of the FAST-PAF configurations.

\begin{tabular}{lc}
\hline Parameter & Quantity \\
\hline Array arrangement & Hexagon \\
Array amount & 217 \\
Element spacing & $0.65 \lambda$ \\
Simultaneous beams & 109 \\
Elements for each beam & 37 \\
\hline
\end{tabular}

into consideration the cost of the beamforming network, the number of the elements is decided to be 9 rings or 217 elements.

In FAST-PAF, the overlapped subarray multibeam technology is applied to reduce the pressure of data processing in beamforming network. According to (16), $d_{3 \text { null }}=0.45 \mathrm{~m}$ at $1.25 \mathrm{GHz}$, and element spacing is $d=0.65 \lambda=156 \mathrm{~mm}$. Therefore, at least 4 rings of elements $(n=4,37$ elements) are necessary to build one subarray and to form a single beam which can cover 95\% energy region of the focal plane. Figure 5(b) shows the sensitivity achieved by 37 elements as the 4-ring beamformed subarray moves from center to the array edge at an element-scale interval, which is illustrated in Figure 5(c). As the location of the subarray moves, the beams steer away from the boresight and overlap which achieve high sensitivity in a wide field of view. In conclusion, Table 3 shows the array configurations of the FAST.

\section{Element Design}

According to the content above, the phased array of the FAST consists of 217 elements and covers a circular area with a radius of $1.3 \mathrm{~m}$. PAFs should be able to work in L-band. Additionally, PAFs should be dual-polarized, have high isolation, and be miniaturized. Dipole-like antennas are identified as candidates because of their low loss and low profile and being easy to array and other good properties. A regular dipole fed by a coaxial balun is a good choice. However, its voltage standing wave ratio is very sensitive to the dimension of the feeding probe. Moreover, the machining accuracy of the probe is not easy to guarantee. In this study, a cross-dipole fed by microstrip baluns is introduced instead of coaxial cross-dipole antenna. Microstrip integrated balun is widely used in the communication and radar systems owing to its salient features such as light weight, wideband, low cost and ease of fabrication, and suitability for integration with circuit modules [17].

In Figure 6, the geometry of the proposed antenna is modeled using Ansoft HFSS. The three pictures on the left side are top and side views of the antenna. And the right two pictures show the feeding structure of balun. The antenna employs two vertical substrates that are cross-arranged and four thick copper discs and a horizontal metalized ground plane is used as a reflector to avoid backward radiation and to achieve high gain. All substrates are designed using the standard Arlon AD450, with a thickness of $1.0 \mathrm{~mm}$. The ground plane is an aluminum plate.

The feeding structure of the antenna is the microstrip balun. Two baluns are designed to feed the dipoles. One balun
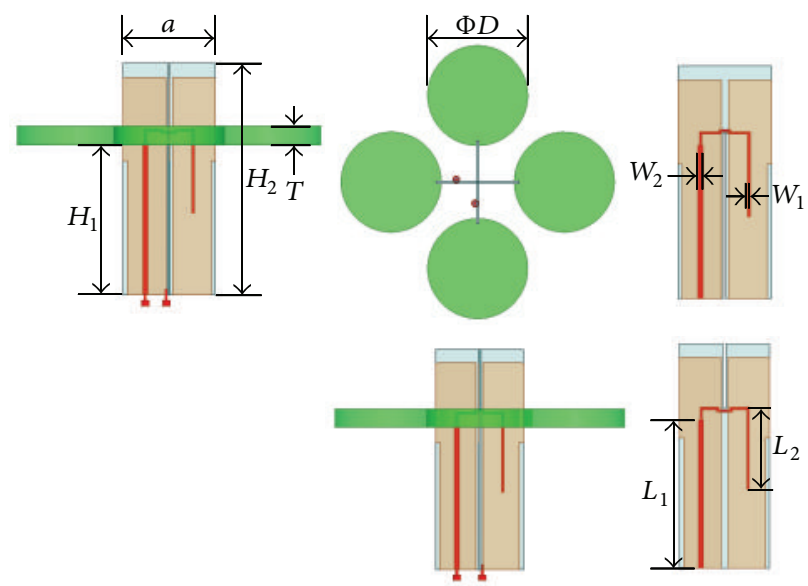

FIGURE 6: Geometry of the proposed antenna.

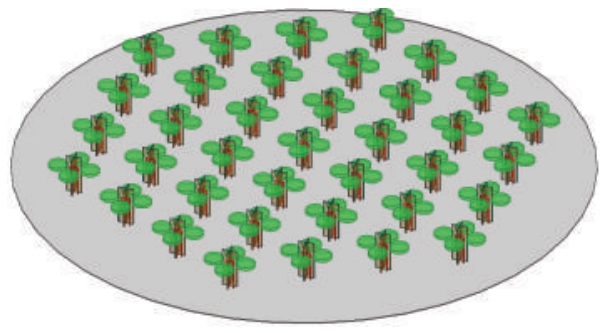

FIGURE 7: A 37-element hexagonal array for FAST.

TABLE 4: Some parameters of the designed antenna.

\begin{tabular}{lccccccccc}
\hline Parameters & $a$ & $H_{1}$ & $H_{2}$ & $T$ & $D$ & $W_{1}$ & $W_{2}$ & $L_{1}$ & $L_{2}$ \\
\hline Values $(\mathrm{mm})$ & 33 & 83.5 & 54 & 7 & 40 & 0.9 & 1.8 & 55 & 30.5
\end{tabular}

is bent down at the middle of the horizontal microstrip line, whereas the other balun is bent up to isolate itself from the former. The two baluns are connected to the feeding probe of a $50 \Omega$ SMA launcher.

Four thick copper discs with rectangular slots on the edges are connected to the substrates as radiation parts. Two of the four discs form the dipole and control one polarization. As ground planes of the microstrip baluns, four vertical rectangular patches located on the opposite sides of the baluns join the arms of the dipoles and the ground plate together. Some of the optimized key parameters of the proposed antenna shown in Figure 6 are listed in Table 4.

A hexagonal array of 37 elements is presented in Figure 7. The array is mounted on an aluminum plate with a radius of $260 \mathrm{~mm}$ and a thickness of $2.0 \mathrm{~mm}$.

\section{Results}

5.1. Element and Array Characteristics. To validate the designs, a prototype of the dual-polarized dipole antenna is fabricated, as shown in Figure 8. The simulated and measured $S$ parameters are depicted in Figure 9(a). The measured reflection coefficients are below $-10 \mathrm{~dB}$ over the operating band from $1.0 \mathrm{GHz}$ to $1.5 \mathrm{GHz}$. Figure 9(b) shows the return 


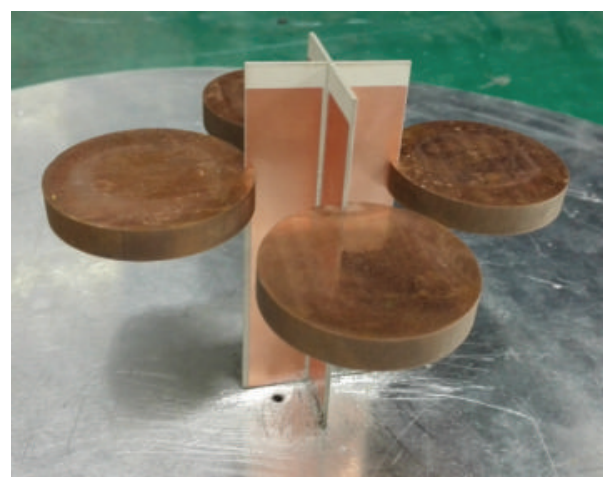

Figure 8: Photo of the cross-dipole.

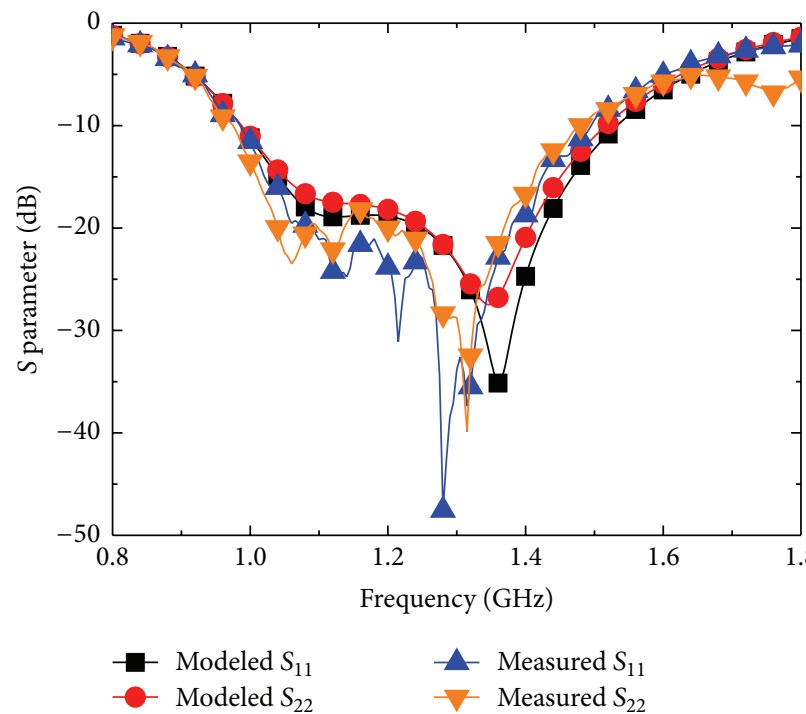

(a)

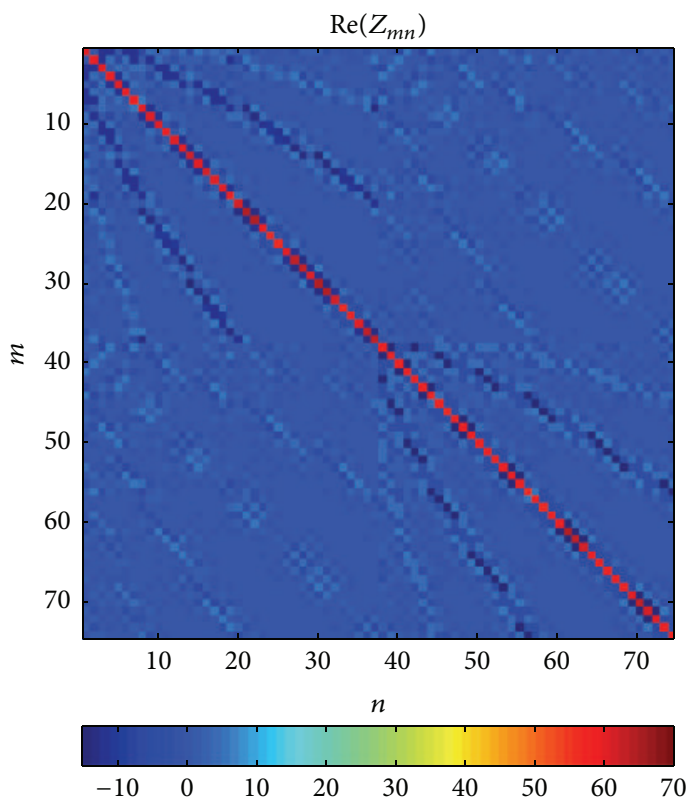

(c)

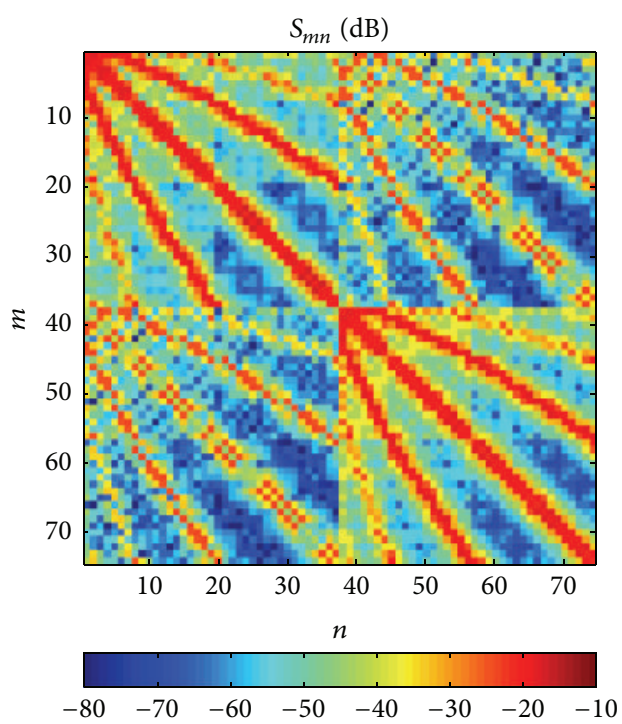

(b)

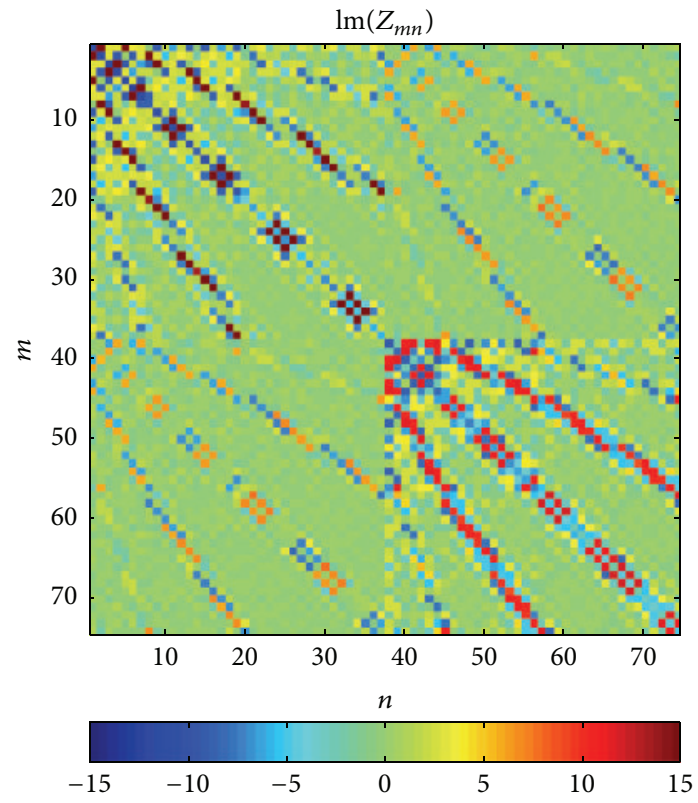

(d)

FIGURE 9: Scattering and impedance characteristic. (a) Modeled and measured reflection coefficients of the antenna. (b) Scattering matrix of the array. (c) Real part of the impedance matrix. (d) Imaginary part of the impedance matrix. 


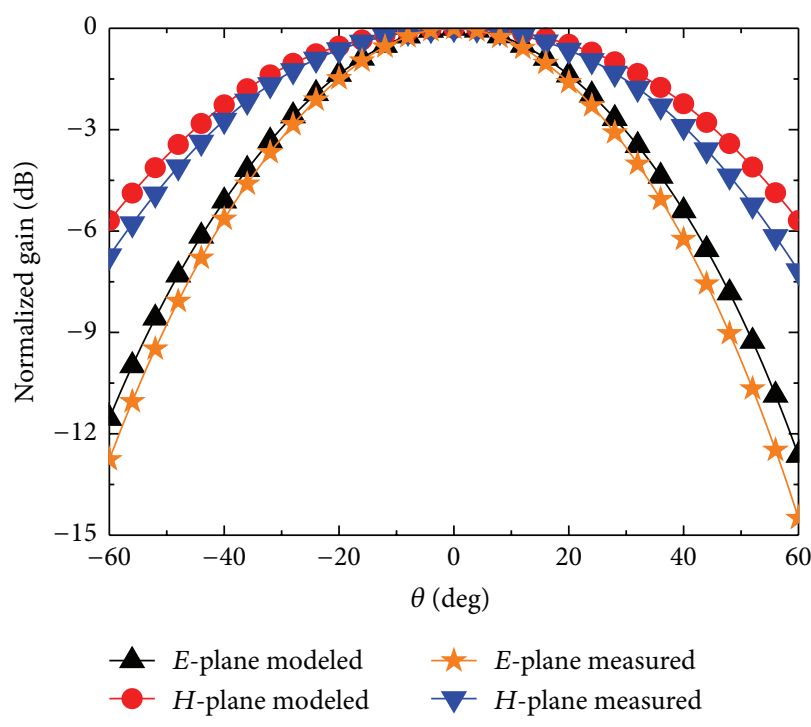

(a)

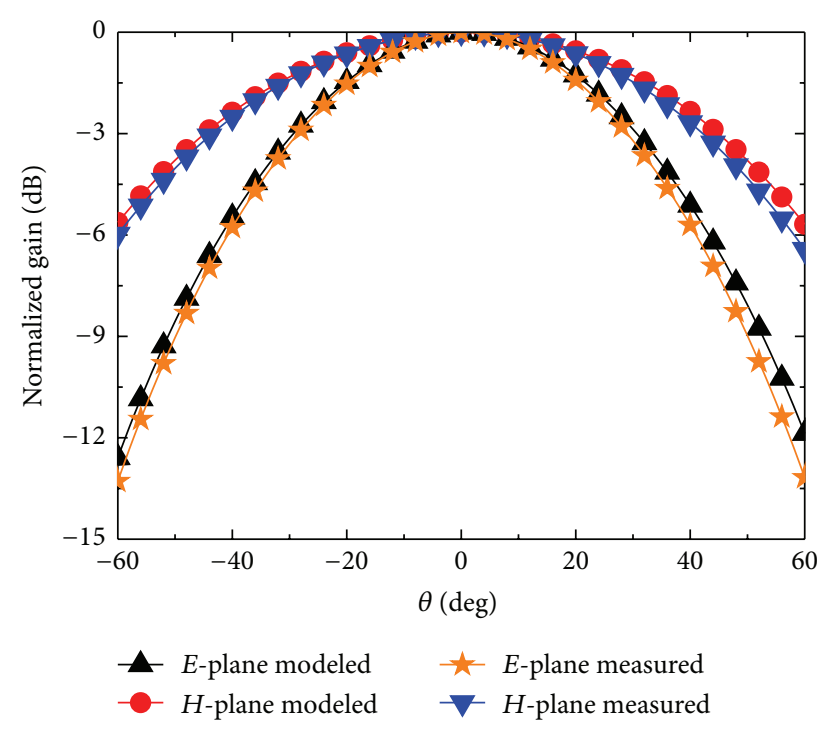

(b)

FIGURE 10: Normalized radiation patterns of the proposed antenna. (a) Radiation pattern with port 1 excited. (b) Radiation pattern with port 2 excited.
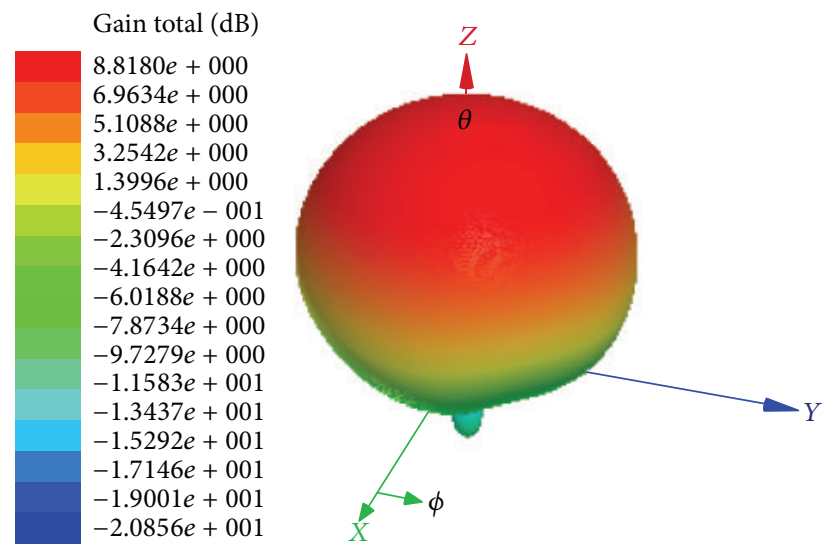

(a)

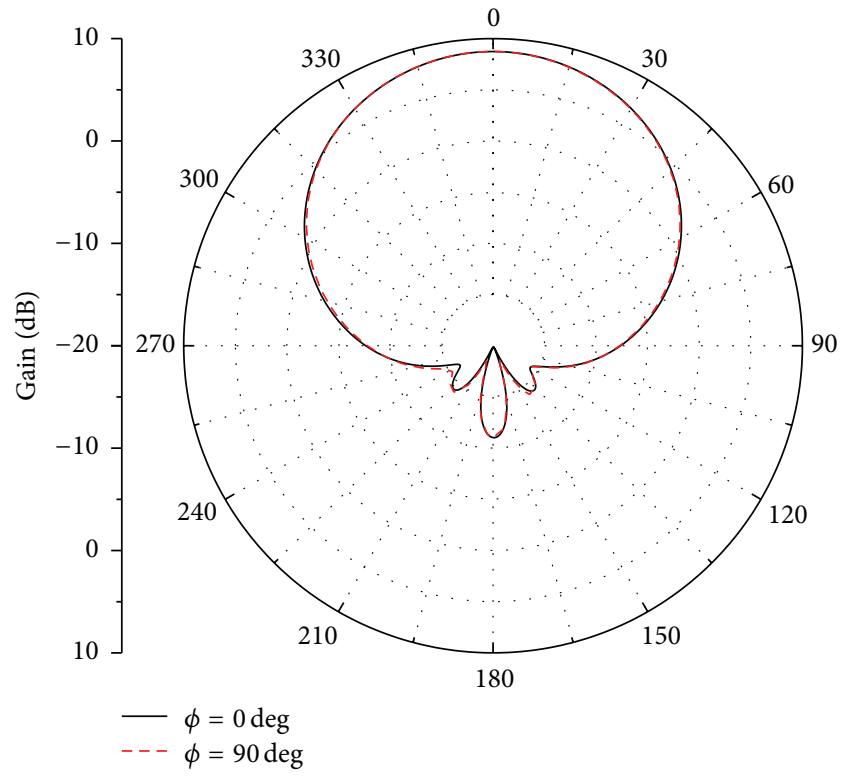

(b)

FIGURE 11: Radiation patterns of the proposed antenna with right-hand circular polarization. (a) 3D polar plot. (b) Polar plot.

and insertion losses of the two-dimensional plot for the 37element array at $1.25 \mathrm{GHz}$. Figures 9(c) and 9(b) describe the impedance characteristics of the antenna with real and imaginary parts of the impedance matrix. These results show that a good impedance match has been realized and high isolation has been achieved.

In terms of a single feed with reflector, when the $-11 \mathrm{~dB}$ width of the normalized radiation pattern is equal to the reflection angle, the antenna efficiency reaches the peak. Given that every radiation pattern of the unit superimposes to form the array pattern and beams can be changed by adjusting the weight vectors, the irradiation level of the single antenna pattern on the reflector edge is not strictly required to be optimal. Figures 10(a) and 10(b) show the normalized measured and modeled radiations with every port excited. As shown in Figure 10, the normalized gain on $E$ plane is about $-12 \mathrm{~dB}$ at $\pm 60 \mathrm{deg}$, which is close to $-11 \mathrm{~dB}$. Figure 11 shows the radiation patterns of the antenna at central frequency. The two ports are fed with $\pi / 2$ rad phase difference to form right-hand circular polarization. The antenna gain is $8.8 \mathrm{dBi}$ at $1.25 \mathrm{GHz}$. Stable antenna gain of over $7 \mathrm{dBi}$ in the operating frequency is obtained as shown in Figure 12. Figure 13 is 


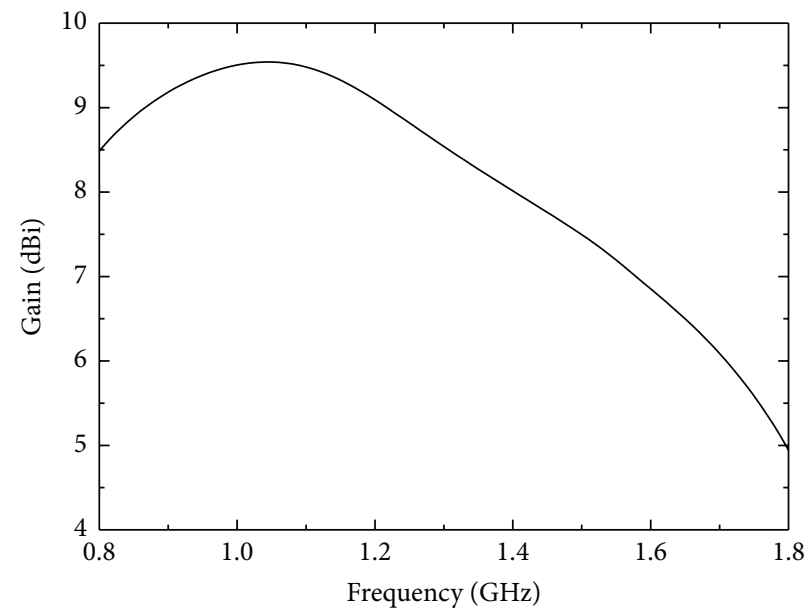

FIGURE 12: Gain versus frequency for a dual-feed circularly polarization.
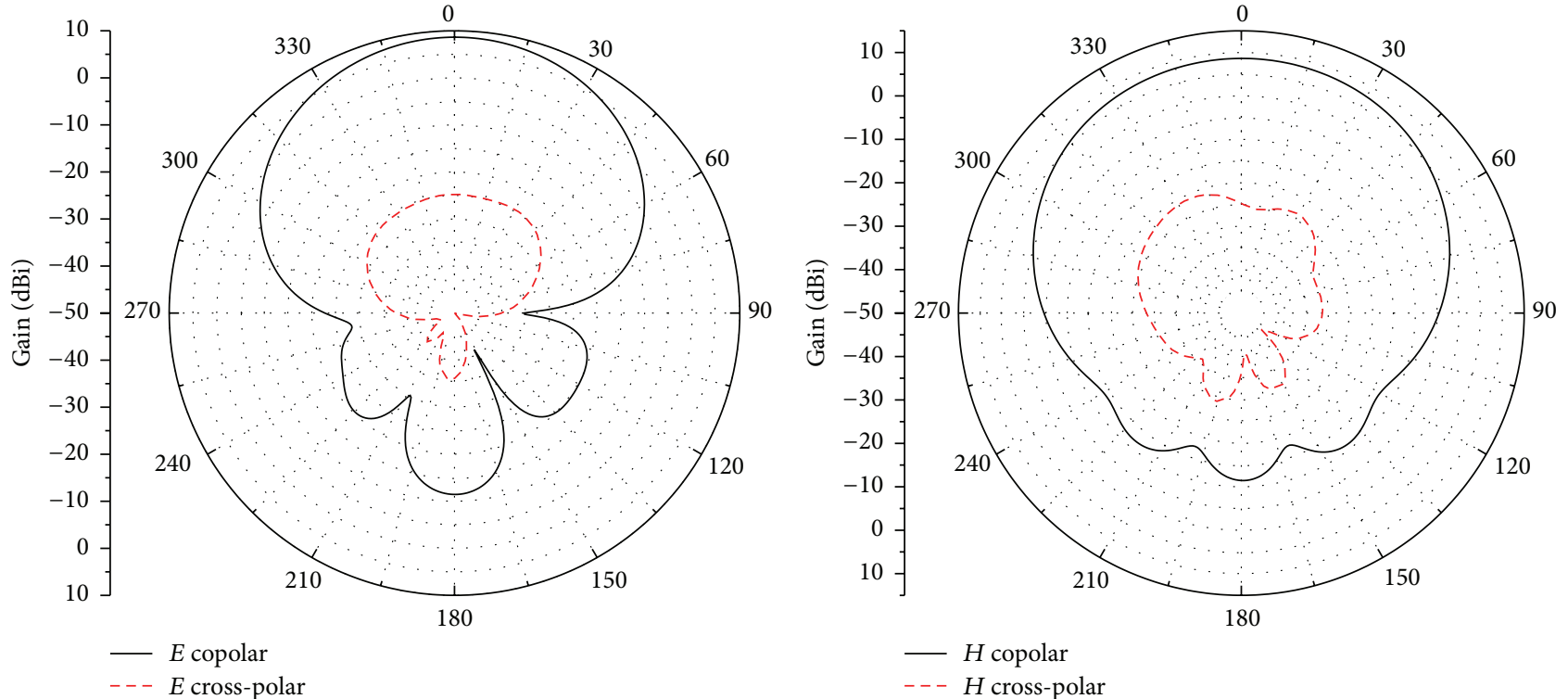

(a)
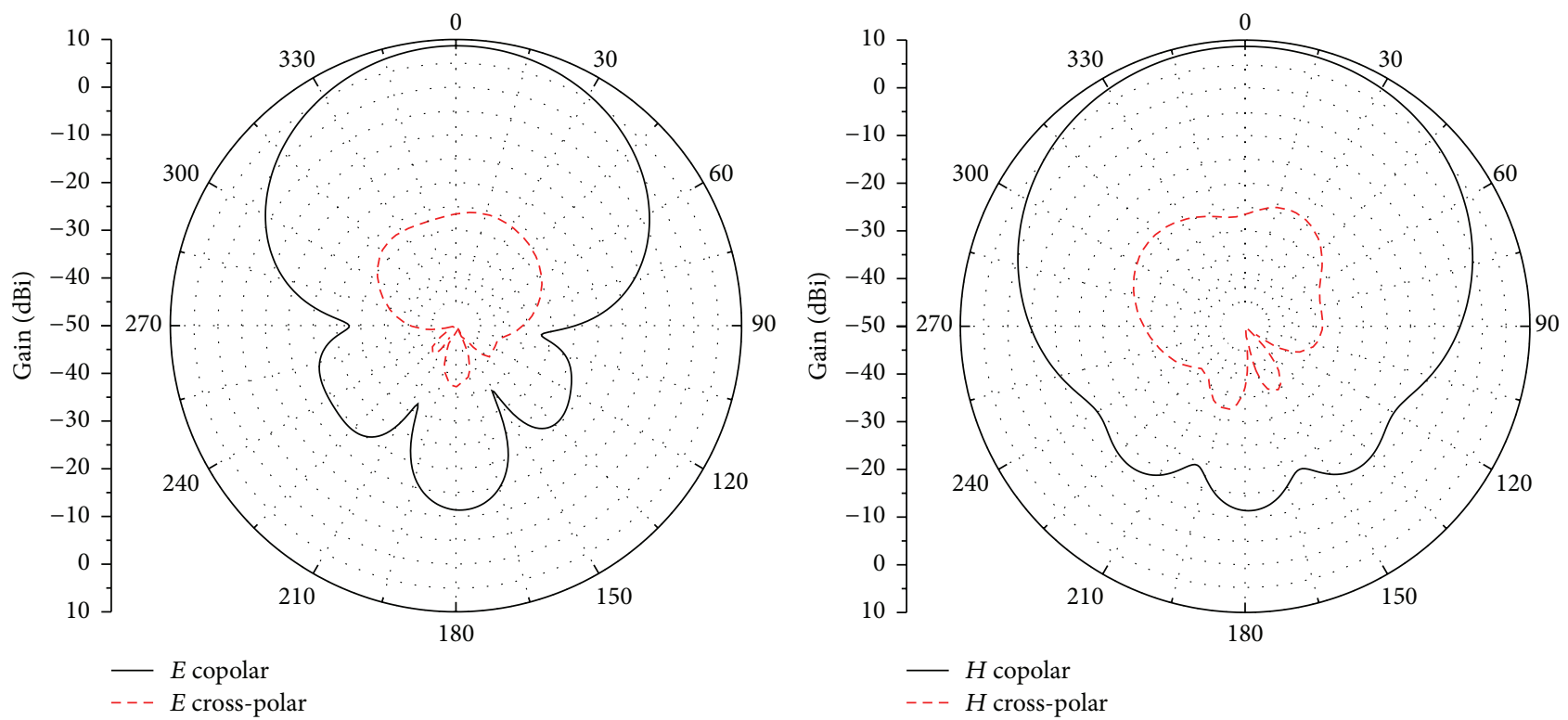

(b)

FIGURE 13: Radiation patterns of primary polarization and cross-polarization for the proposed antenna. (a) Only port 1 is excited. (b) Only port 2 is excited. 


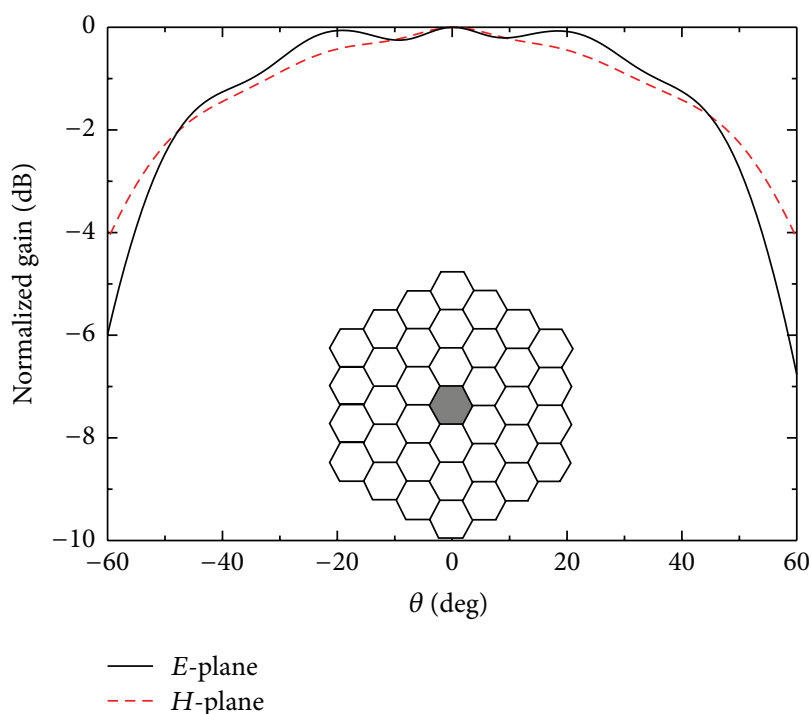

(a)

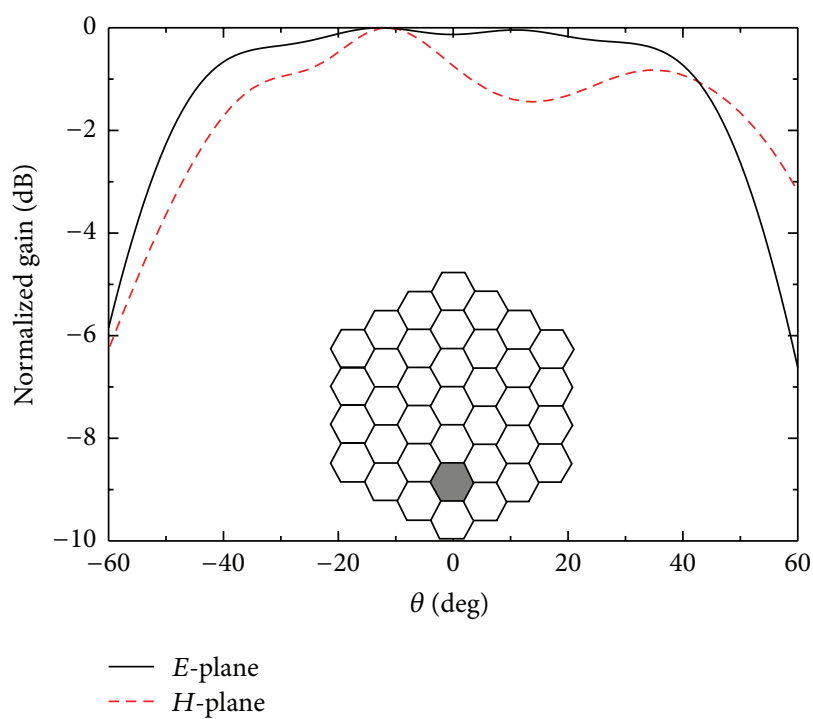

(c)

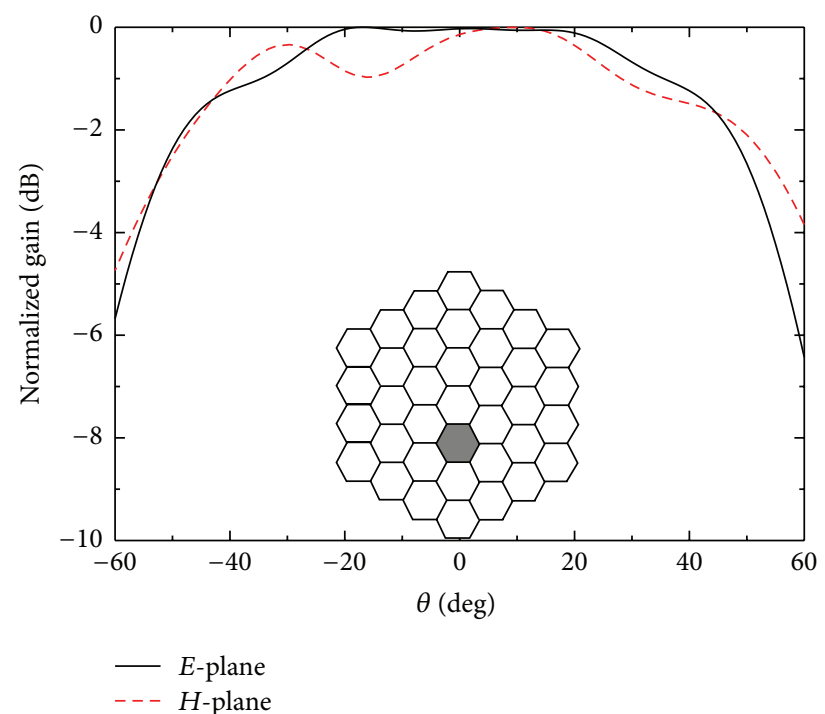

(b)

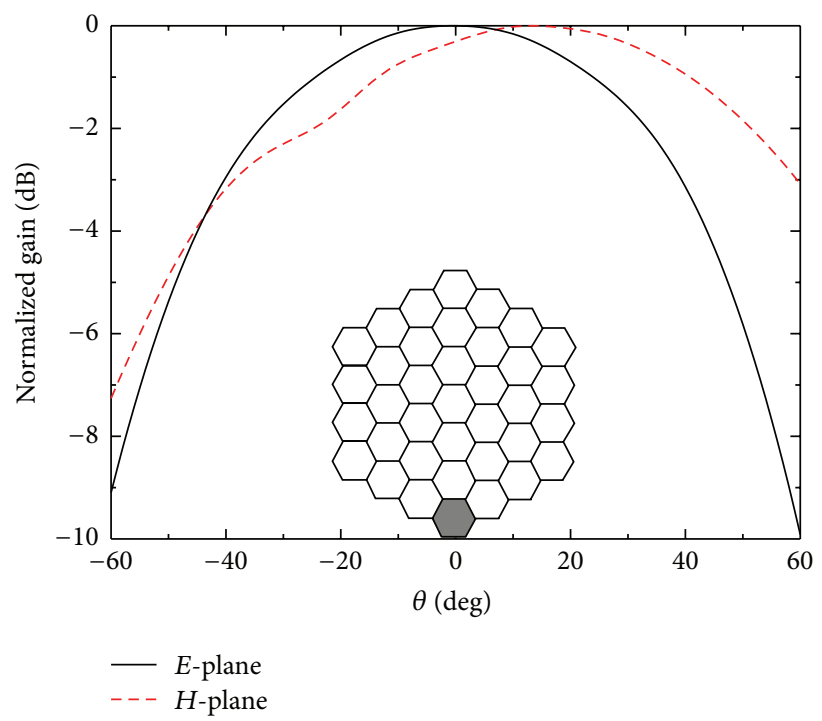

(d)

FIGURE 14: Normalized radiation patterns of one element in different positions of the array with linear polarization. (a) Central position 1. (b) Position 2. (c) Position 3. (d) Position 4.

the radiation patterns of primary polarization and crosspolarization for the proposed antenna at $1.25 \mathrm{GHz}$. The results show that low cross-polarization level is achieved. Figure 14 shows the normalized radiation patterns of one element in different positions of the array with linear polarization. Radiation efficiency of the antenna is $98.99 \%$ at the central frequency $1.25 \mathrm{GHz}$, which is over $98 \%$ and meets the criteria in Table 2.

5.2. System Performance. The evaluation criterion of this design is system performance. Given that receiver noise is the major contributor to overall system noise temperature and the LNA gain is quite large at $40 \mathrm{~dB}$, LNA noise significantly dominates system noise. Thus a liquid heliumcooled (cryocooled) LNA is applied to reduce noise [7]. A $300-\mathrm{m}$ aperture is adopted to estimate the achievable
TABLE 5: Parameters for the LNAs.

\begin{tabular}{lc}
\hline LNA parameters & Quantity \\
$(1.25 \mathrm{GHz})$ & $6.29 \mathrm{~K}$ \\
\hline$T_{\min }$ & $71.2+j 15.7 \Omega$ \\
$Z_{\text {opt }}$ & $0.706 \Omega$ \\
$R_{N}$ & $39.2 \mathrm{~dB}$ \\
$G_{\mathrm{LNA}}$ & \\
\hline
\end{tabular}

sensitivity of the FAST reflectors. The parameters of this LNA are illustrated in Table 5, where $T_{\min }$ is the minimum noise temperature of the amplifier, $Z_{\text {opt }}$ is the optimal signal source impedance of the LNA, $R_{N}$ is the equivalent noise resistance of the amplifier, and $G_{\mathrm{LNA}}$ is the LNA gain. Figure 15 shows the simulation results with room temperature and with a 


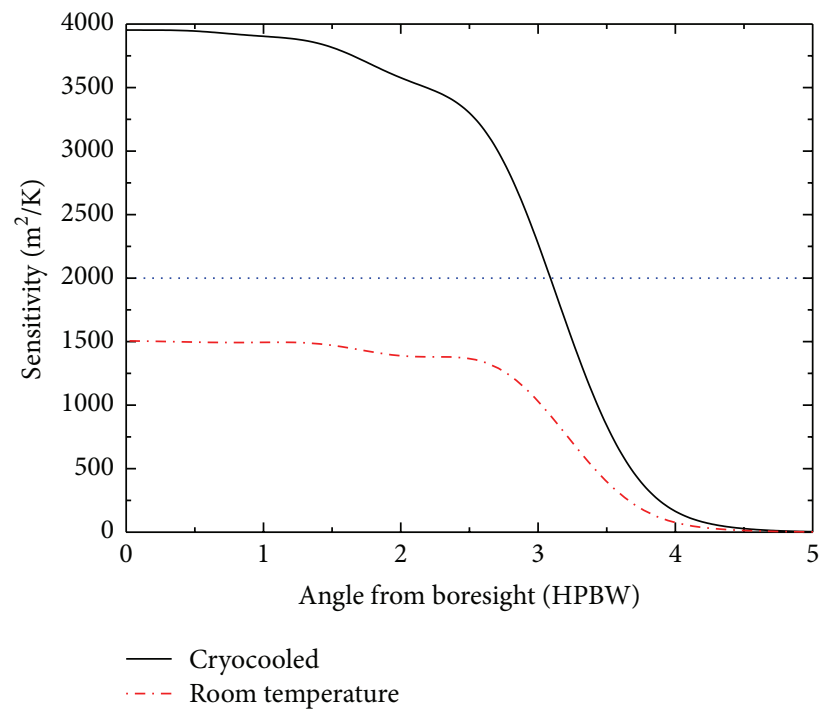

(a)

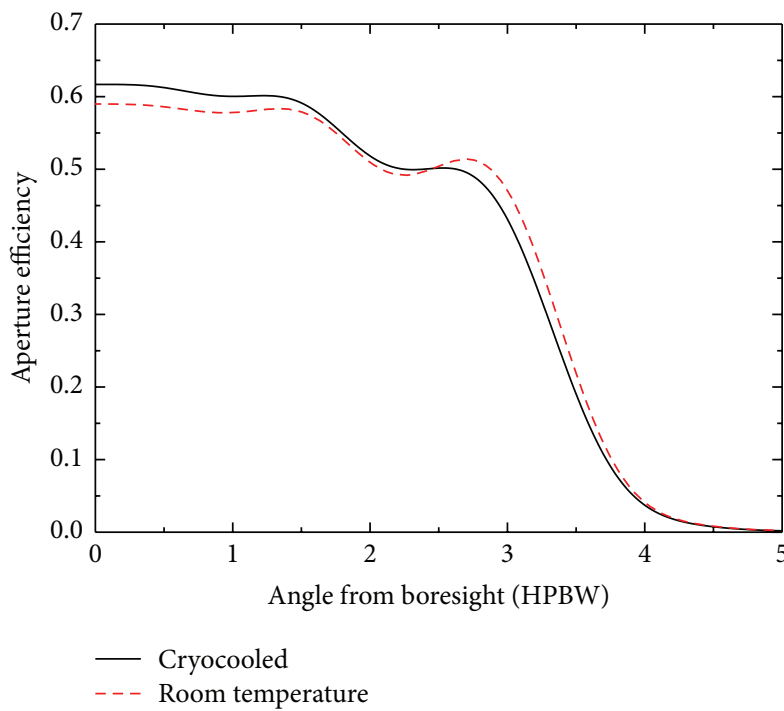

(b)

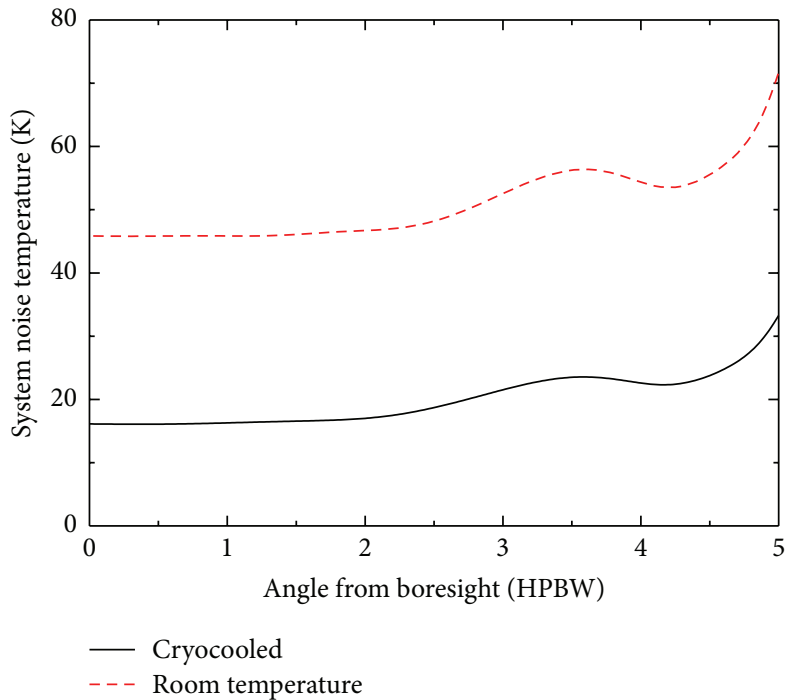

(c)

FIGURE 15: System performance of the 37-element PAF with room temperature and cryocooled LNA. (a) Sensitivity. (b) Aperture efficiency. (c) System noise temperature.

TABLE 6: Parameters of system performance.

\begin{tabular}{lc}
\hline Parameters & Quantity \\
\hline Sensitivity & $3953 \mathrm{~m}^{2} / \mathrm{K}$ \\
System noise temperature & $16.1 \mathrm{~K}$ \\
Aperture efficiency & $61.7 \%$ \\
Field of view $\left(\mathrm{HPBW}^{2}\right)$ & $3.09 \times 3.09$ \\
\hline
\end{tabular}

cryogenic system for LNA including sensitivity, aperture efficiency, and noise temperature of the dual-polarized array. The overall system performance index is shown in Table 6 .

As shown in Figure 15(a), sensitivity increases significantly with cooled LNA, and high sensitivity is achieved far beyond the requirement (the horizontal dotted line in the plot) over a slice of the field of view. The two curves of aperture efficiency are similar in Figure 15(b). Figure 15(c) shows that, with cryocooled LNAs, system noise temperature has reduced a lot and has stable distribution which is less than $20 \mathrm{~K}$ over a wide field of view.

\section{Conclusion}

In this study, a broadband dual-polarized antenna for the FAST-PAF is designed and a single element is manufactured. The simulated and measured results of the fabricated element verify the design. An array of 37 elements has been modeled. The system performance of the PAF satisfies the requirement of the FAST. The cross-dipole antenna is suitable for the next 
generation radio telescopes. The next step in this research will be to cooperate with the FAST team of National Astronomical Observatories of China and integrate the fabricated array elements and cryostat for on-reflector tests.

\section{Abbreviations}

FAST: Five-Hundred-Meter Aperture Spherical Telescope

PAF: $\quad$ Phased array feed

LNA: Low noise amplifier

SNR: Signal-to-noise ratio

HPBW: Half-power beam width.

\section{Competing Interests}

The authors declare that there is no conflict of interests regarding the publication of this paper.

\section{Acknowledgments}

This study is supported in part by the National Natural Science Foundation of China (no. 11273017).

\section{References}

[1] R. Anwar, M. T. Islam, N. Misran, G. Gopir, B. Yatim, and M. F. Asillam, "Effect of parasitic element on $408 \mathrm{MHz}$ antenna for radio astronomy application," International Journal of Antennas and Propagation, vol. 2014, Article ID 614952, 4 pages, 2014.

[2] B. Veidt, T. Burgess, R. Messing, G. Hovey, and R. Smegal, "The DRAO phased array feed demonstrator: recent results," in Proceedings of the 13th International Symposium on Antenna Technology and Applied Electromagnetics and the Canadian Radio Science Meeting (ANTEM/URSI '09), pp. 1-4, IEEE, Banff, Canada, February 2009.

[3] A. Chippendale, J. O’Sullivan, J. Reynolds et al., "Phased array feed testing for astronomy with ASKAP," in Proceedings of the IEEE International Symposium on Phased Array Systems and Technology (ARRAY '10), pp. 648-652, IEEE, October 2010.

[4] K. F. Warnick, D. Carter, T. Webb et al., "Towards a high sensitivity cryogenic phased array feed antenna for the Green Bank Telescope," in Proceedings of the 30th URSI General Assembly and Scientific Symposium, pp. 1-4, Istanbul, Turkey, August 2011.

[5] R. Nan, "Five hundred meter aperture spherical radio telescope (FAST)," Science in China, Series G: Physics Astronomy, vol. 49, no. 2, pp. 129-148, 2006.

[6] Y. Wu, K. F. Warnick, and C. Jin, "Design study of an Lband phased array feed for wide-field surveys and vibration compensation on FAST," IEEE Transactions on Antennas \& Propagation, vol. 61, no. 6, pp. 3026-3033, 2013.

[7] D. E. Carter, Active impedance matching and sensitivity optimized phased array deed design for radio astronomy [M.S. thesis], Brigham Young University, Provo, Utah, USA, 2011.

[8] C. K. Hansen, K. F. Warnick, B. D. Jeffs, J. R. Fisher, and R. Bradley, "Interference mitigation using a focal plane array," Radio Science, vol. 40, no. 5, pp. 453-468, 2005.

[9] D. Jones, Characterization of a phased array feed model [M.S. thesis], Brigham Young University, Provo, Utah, USA, 2008.
[10] B. D. Jeffs, K. F. Warnick, J. Landon et al., "Signal processing for phased array feeds in radio astronomical telescopes," IEEE Journal on Selected Topics in Signal Processing, vol. 2, no. 5, pp. 635-646, 2008.

[11] J. C. Landon, Development of an experimental phased array feed system and algorithms for radio astronomy [Ph.D. thesis], Brigham Young University, Provo, Utah, USA, 2011.

[12] C. K. Hansen, Beamforming techniques and interference mitigation using a multiple feed array for radio astronomy [M.S. thesis], Brigham Young University, Provo, Utah, USA, 2004.

[13] D. P. Petersen and D. Middleton, "Sampling and reconstruction of wave-number-limited functions in $\mathrm{N}$-dimensional euclidean spaces," Information \& Control, vol. 5, no. 62, pp. 279-323, 1962.

[14] R. A. Monzingo, R. L. Haupt, and T. W. Miller, Optimum Array Processing. Introduction to Adaptive Arrays, IET Digital Library, 2011.

[15] Y. Lo, "On the beam deviation factor of a parabolic reflector," IRE Transactions on Antennas and Propagation, vol. 8, no. 3, pp. 347-349, 1960

[16] Y. Wu, B. Du, X. M. Liu, and C. J. Jin, "Effect of feed array configuration on the field-of-view of radio telescope antenna," Journal of Electronics, vol. 30, no. 4, pp. 362-367, 2013.

[17] O. B. Linton and Z. Xiao, "Suppression of cross-polarization of the microstrip integrated balun-fed printed dipole antenna," International Journal of Antennas \& Propagation, vol. 2014, Article ID 765891, 8 pages, 2014. 


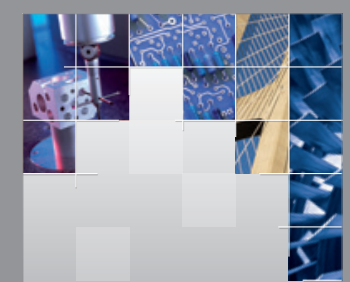

\section{Enfincering}
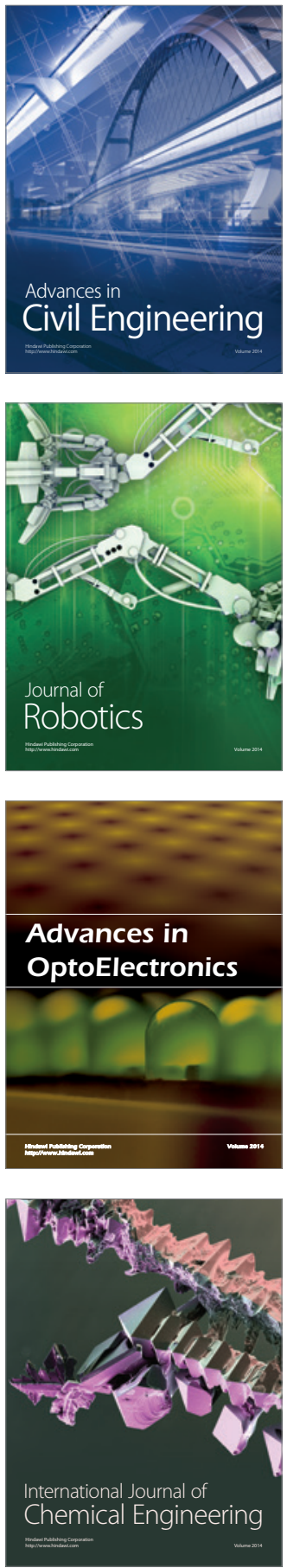

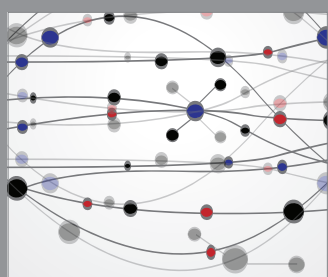

The Scientific World Journal

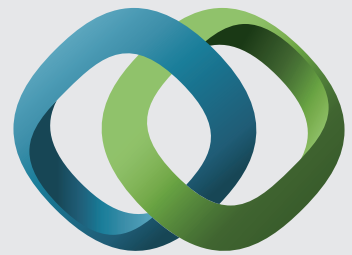

\section{Hindawi}

Submit your manuscripts at

http://www.hindawi.com
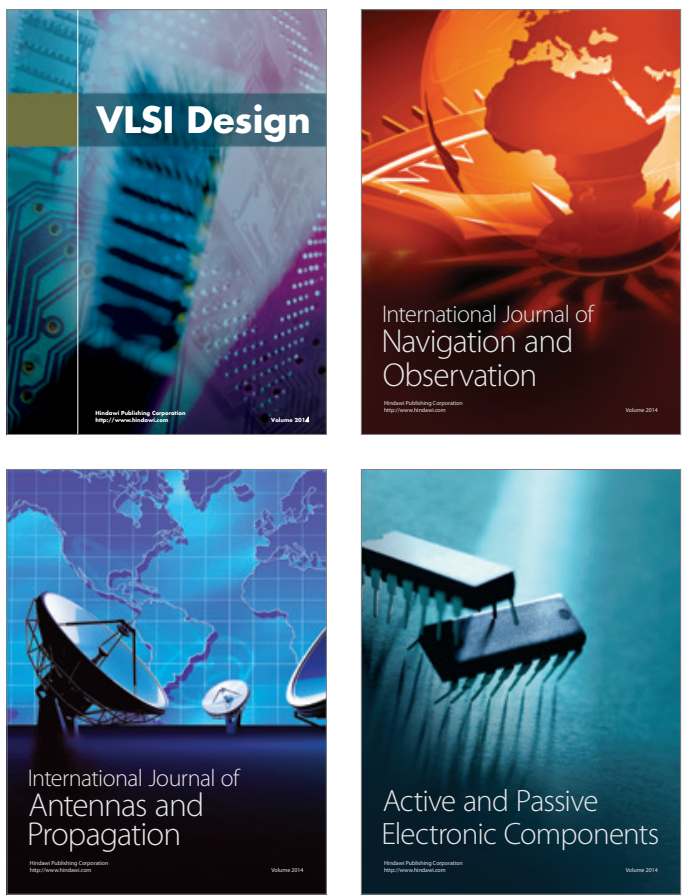
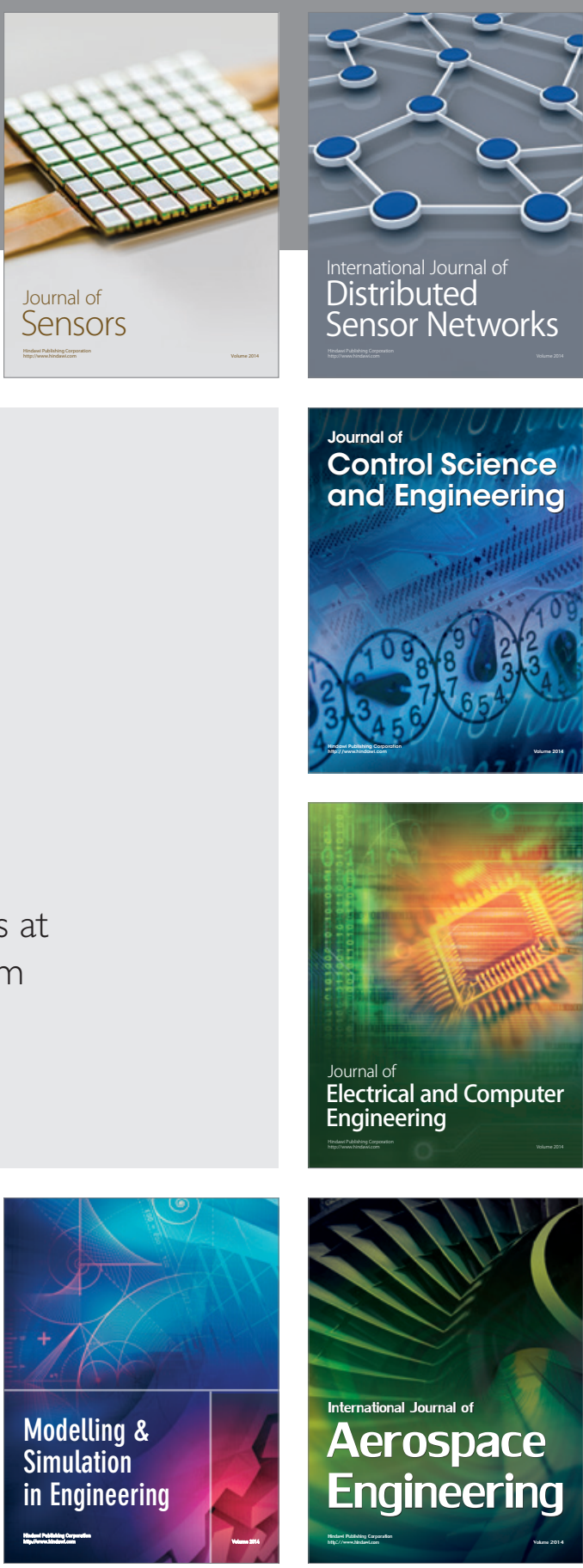

International Journal of

Distributed

Sensor Networks

Journal of

Control Science

and Engineering
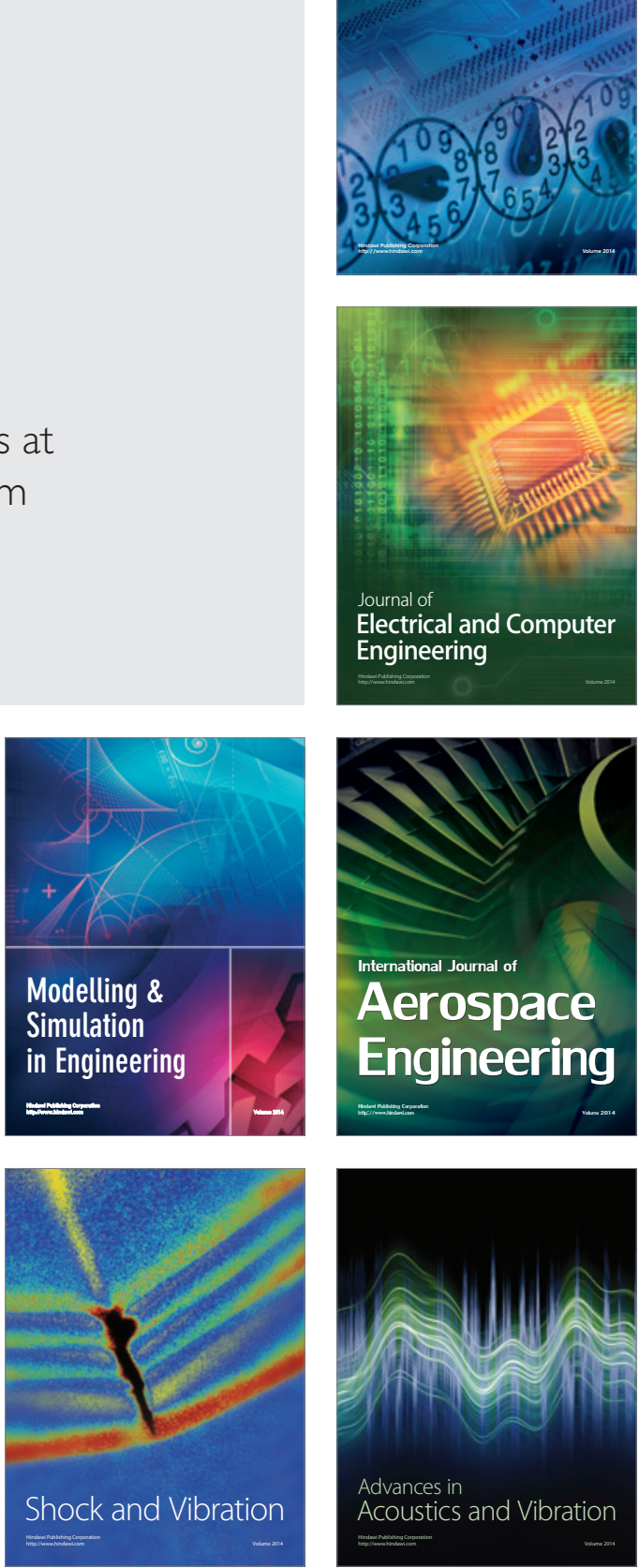